\title{
Exploring the Potential of VCSEL Technology for Agile and High Capacity Optical Metro Networks \\ [Invited]
}

\author{
Michela Svaluto Moreolo, Josep M. Fàbrega, Laia Nadal, and F. Javier Vílchez \\ Optical Networks and Systems Dept., Communication Networks Division, \\ Centre Tecnològic de Telecomunicacions de Catalunya (CTTC/CERCA) \\ Castelldefels, Spain \\ michela.svaluto@cttc.es
}

\begin{abstract}
In this paper, vertical cavity surface emitting laser (VCSEL) technology is presented as potential prominent performer to address key challenges and novel functionalities of future optical metro networks. The adoption of VCSEL-based modules is particularly attractive for the implementation of programmable (SDN-enabled) transceiver architectures, targeting a radical reduction of cost, power consumption and footprint. Different flavours of these architectures are presented to enable agile, scalable and high capacity optical metro networks. Furthermore, advanced functionalities and programmability aspects are analysed to explore the potential of adopting solutions based on this technology.
\end{abstract}

Keywords- vertical cavity surface emitting laser (VCSEL); optical metro networks; transceiver architecture; sliceable bandwidth variable transceiver (S-BVT); defragmentation.

\section{INTRODUCTION}

The future optical metro network scenario is evolving towards a highly-dynamic paradigm where multiple, new and bandwidth-hungry $5 \mathrm{G}$ services should be supported, minimizing the cost and power consumption. As an example, it is envisioned that content delivery networks (CDNs) will carry $71 \%$ of Internet traffic by 2021 (up from 52\% in 2016) and that 35\% of end-user Internet traffic will be delivered within a metro network by 2021 (22\% in 2016) [1]. Actually, CDNs will carry traffic closer to the end user and, although at present much CDN traffic is onto regional core networks, the metro-delivered traffic is growing faster than core-delivered traffic according to the predicted percentage increase [1]. Thus, future optical metro networks should be able to accommodate the envisioned increase of speed and volume of traffic, as well as low-rate service connections, while managing adaptive bit rates and peak of traffic according to the available bandwidth.

In this context, programmable adaptive transceivers supporting great capacity and dynamicity arise as key enabler to address these challenges, such as offering ultrabroadband $5 \mathrm{G}$ services and managing high peak-average traffic ratio. Particularly, bitrate and bandwidth variable transceivers (BVTs) allow software defined optical transmission adaptive to different network conditions and requirements. Sliceable BVTs (S-BVTs), supporting multi-flow transmission, are able to give service to a higher number of sites and to provide greater capacity per site, resulting a crucial element for supporting any type of traffic within an evolutionary metro network scenario. Novel programmable - to be integrated in a software defined network (SDN) based control plane - transceiver architectures, transparent to service and with a wide range of granularities, are required to facilitate metro network operation, supporting increased bandwidth demand and network dynamicity. A modular design is also desirable for targeting scalable architectures allowing to grow-as-needed. Furthermore, a cost-effective implementation should be targeted to be suitably adapted to the metro segment needs.

Cost-effective data plane solutions for flexible metro networks using S-BVTs have been proposed in [2]. They are based on orthogonal frequency division multiplexing (OFDM) technology. Actually, multicarrier modulation (MCM), as OFDM or discrete multitone (DMT), enables dynamic and flexible adaptation to traffic/channel conditions with fine granularity, when bit and power loading (BL/PL) algorithms are implemented at the digital signal processing (DSP) [3]. Alternative architectures for metro/regional scenario have been assessed and analyzed also from a techno-economic point of view in [4]. It has been shown that multiple low bit rate flexgrid connections can be supported over regional optical paths, adopting centralized SBVTs shared among multiple end-points with cost-effective transceiver schemes. In [5] transparent/dynamic delivery of mobile front-/back-haul in a converged optical metro architecture has been experimentally demonstrated, employing SDN-enabled S-BVTs based on adaptive MCM. The proposed architecture is specifically tailored for a transparent services delivery across the access and metro network segments. Network testbed 
experiments show successful connectivity at distances up to 175 $\mathrm{km}$ and capacities beyond $30 \mathrm{~Gb} / \mathrm{s}$ per flow.

In line with these previous works, we propose to leverage the concept of S-BVT to design and implement modular transceiver architectures able to support the evolutionary scenario of future metro networks, considering a centralized S-BVT of highcapacity (e.g. able to support high peak rate) shared among distributed (S)-BVTs of lower capacity. More precisely, S-BVTs would be located at the metro/aggregation nodes, either metro/regional or metro/access (exchange) nodes, suitably sizing the number of modules and the slice-ability according to the need; while simpler and even more cost-effective BVT architectures are located closer to the end-user.

In this framework, energy- and cost-efficient photonic technologies should be explored to design and implement novel modular S-BVT architectures, to meet the requirements of the metro segment, while providing high capacity [6]. Particularly, in order to reduce the transmitter cost, direct modulation of the laser source can be considered instead of using external modulation. The adoption of vertical cavity surface emitting diode (VCSEL) technology could be considered as an attractive option to provide energy-efficient, low-cost solutions with small footprint. In fact, VCSEL manufacturing cost is substantially lower than other common options (e.g. DFB lasers), and the transceiver devices can be integrated in the same photonic platform. Thus, this technology is worth to be explored for the design and implementation of S-BVTs especially tailored for the metro segment [7].

VCSEL technology is usually considered for short-reach and low-data-rate applications at $850 \mathrm{~nm}$. Highest bitrates have been achieved adopting 4-PAM, carrierless-amplitude-phase (CAP) modulation and DMT modulation [8]. Coherent detection has been proposed to flexibly extend the achievable reach, showing the potential of VCSELs for $100 \mathrm{G}$ and beyond metro network applications at $1550 \mathrm{~nm}$ and using polarization division multiplexing [9]. DMT, as OFDM, enables spectral manipulation with very fine granularity (subwavelength level), and is considered a promising candidate to support future 1T-class transceivers [10].

Actually, MCM with BL/PL enables spectral manipulation, with spectrum granularity even finer than elastic optical networks (EONs) $(12.5 \mathrm{GHz})$ such as ultra-dense wavelength switching networks [11]. Flexible transport, with subwavelength granularity and variable capacity per channel, enables optimal resource usage and advanced functionalities [3]. Particularly, this allows a soft migration of fixed grid networks towards a flexible architecture and also enables a subcarrier-based defragmentation of the spectrum, without requiring a network re-optimization [3].

In this paper, VCSEL technology is explored to design the fundamental building block and/or specific module(s) of the (S)BVT, as well as to implement advanced functionalities, which can be particularly suitable for future optical metro networks.

The paper is organized as follows. In Sec. II, different flavors of programmable S-BVT architectures adopting VCSEL-based modules are presented to enable agile, scalable, cost-effective and high capacity optical metro network. Different types of VCSEL are introduced and their capabilities presented/discussed, reporting recent results. Sec. III deals with advanced functionalities enabled by programmable transceivers equipped with VCSELs; Sec. III A is particularly devoted to the capability of mitigating the spectrum fragmentation. Conclusions are drawn in Sec IV, where future work on this topic is also discussed.

\section{Programmable Transceiver Architectures Adopting VCSEL TECHNOLOGY}

Different architectures and modulation formats can be used to implement S-BVTs and programmable transceivers [12]. Coherent transceivers combined with suitable DSP and advanced complex modulation formats provide high capacity/reach and

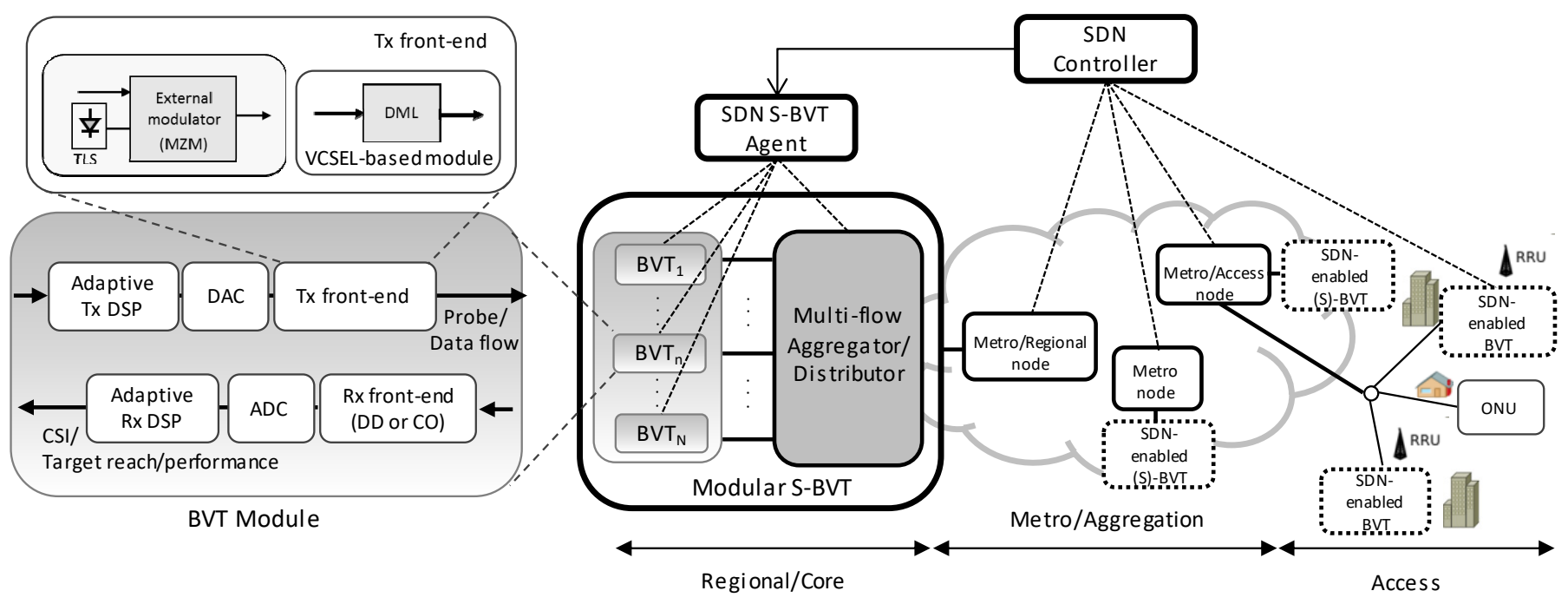

Fig. 1. Schematic architecture of an SDN-enabled modular S-BVT to be suitably sized according to the network/node needs and related scenario. 
flexibility at the expense of cost and energy-efficiency, which can result not appropriate/affordable for the metro segment. Alternative approaches more suitable and specifically tailored for metro networks combine simple intensity or amplitude modulation at the transmitter, while adopting direct detection (DD) or keeping coherent (CO) detection at the receiver $[3,4,6]$.

As mentioned in Sec. I, a VCSEL-based transmitter scheme can be combined with either a simple low-cost detection scheme based on DD $[8,13]$ or $\mathrm{CO}$ detection to achieve ultimate performance in terms of reach and capacity [9].

To address very high capacity link target (e.g. up to 100 $\mathrm{Tb} / \mathrm{s}$ ), both spectrum and space dimensions should be considered [14-15].

Figure 1 shows a schematic architecture relying on a modular approach to allow suitably sizing the S-BVT according to the network needs or to the specific node, where the (S)-BVT is located. The programmable (SDN-enabled) S-BVT consists of an array of $N$ BVT modules and an aggregator/distributor of the multiple flows (slices). This aggregation/distribution element can be implemented with a bandwidth-variable spectrum selective switch (SSS). The multiple slices are transmitted over the network as a single high-capacity flow or can be split into lower capacity flows routed towards independent paths to reach different destination nodes. Alternative choices for the transceiver optoelectronic front-ends are indicated in Fig. 1. Direct modulation of the laser source (DML), using a VCSELbased module, at the BVT transmitter (Tx), is proposed instead of adopting external modulation (e.g. using a Mach Zehnder modulator, MZM) with a tunable laser source (TLS). Tunable VCSEL modules can be also envisioned. The adaptive DSP of the BVT array allows implementing DMT or OFDM, including margin adaptive (MA) or rate adaptive (RA) BL/PL algorithms, to suitably adapt the transmission at a target capacity over a certain reach, according to the channel state information (CSI) and the variable rate request ensuring a target performance. The CSI, and thus the information related to the signal to noise ratio (SNR) corresponding to each DMT/OFDM subcarrier, is retrieved at the receiver, transmitting a probe signal with uniform loading (e.g. adopting 4-QAM format for each subcarrier).

Recently, VCSELs potential has been shown at $1550 \mathrm{~nm}$ with advanced modulation formats for passive optical networks (PONs) and metro/access EONs [16-17]. Particularly, in [17], transparent service delivery at variable data rates has been demonstrated in an SDN converged elastic metro/access optical network with cost-effective programmable transceiver based on VCSEL technology. In that experiment, a directly modulated VCSEL of $4.5 \mathrm{GHz}$ bandwidth working at $\lambda=1539.61 \mathrm{~nm}$ was used for implementing the transmitter module. Up to $16 \mathrm{~Gb} / \mathrm{s}$ with $9 \mathrm{GHz}$ maximum spectral occupation was achieved, adopting DMT with $\mathrm{BL} / \mathrm{PL}$ and $\mathrm{DD}$, considering a minimum power budget of $20 \mathrm{~dB}$ in the access segment. A target bit error ratio (BER) of $4.62 \cdot 10^{-3}$, corresponding to a hard decision forward error correction (HD-FEC) of $7 \%$ overhead, has been taken into account. The performance has been assessed in the ADRENALINE testbed network, shown in Fig. 2. Specifically, it consists of four nodes - two OXCs (optical cross-connects) and two ROADMs (reconfigurable optical add-drop multiplexers) and five amplified links ranging from $35 \mathrm{~km}$ to $150 \mathrm{~km}$ [18]. The $35 \mathrm{~km}$ link between OXC-2 and ROADM-1 is flexgrid as the nodes are equipped with programmable SSS modules. A maximum transmission distance of $200 \mathrm{~km}$ has been successfully covered, considering $50 \mathrm{~km}$ PON tree and a $150 \mathrm{~km}$ single hop path of the ADRENALINE network. The achieved rate was about $8 \mathrm{~Gb} / \mathrm{s}$ (half of the maximum rate) at the target BER.
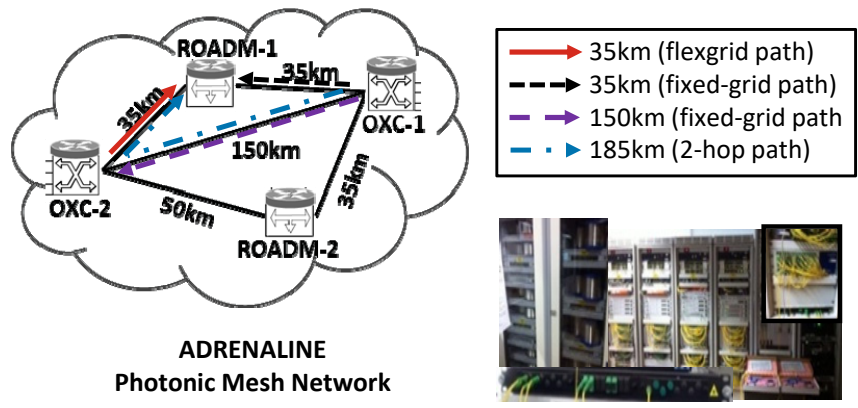

Fig. 2. ADRENALINE network paths and testbed picture.

Adopting larger bandwidth VCSELs and high-performance digital-to-analog and analog-to-digital converters (DAC/ADC), higher capacities can be achieved. A single VCSEL with 3-dB bandwidth of $15 \mathrm{GHz}$, directly modulated with DMT and using $\mathrm{DD}$, has been demonstrated for short-reach applications at $100 \mathrm{~Gb} / \mathrm{s}$ [8]. Up to $95 \mathrm{~Gb} / \mathrm{s}$ (net data rate of $79.2 \mathrm{~Gb} / \mathrm{s}$ ) has been achieved over $4 \mathrm{~km}$ of standard single mode fiber (SSMF). If CO detection is considered, the achievable reach can be further extended to target the metro/regional segment [15]. An S-BVT architecture based on this approach can be implemented integrating a bank of VCSELs at different operating wavelengths. The multiple modules can be suitably enabled/disabled for wavelength selection and bandwidth-variable adaptation.

Widely tunable micro-electro-mechanical system (MEMS) VCSELs allow adapting the operating wavelength over a spectrum range of more than $60 \mathrm{~nm}$ [19]. This type of VCSELs, with 3-dB bandwidth of $7 \mathrm{GHz}$, was successfully demonstrated for DMT transmission at $26 \mathrm{~Gb} / \mathrm{s}$ over $40 \mathrm{~km}$ of SSMF, targeting converged WDM-PON applications [16].

This VCSEL technology can be further explored for implementing the building block or specific modules of an SBVT able to cover extended reach, targeting metro networks [7]. To this extent, single sideband (SSB) OFDM modulation is required to make the direct modulated signal more robust against chromatic dispersion. Thus, a bandwidth variable optical filter, which can be implemented at the S-BVT aggregator stage, should be used. If the network nodes are equipped with suitable SSS, the optical filtering could be performed at any node, not only at the sliceable transceiver. 
The capacity performance of a BVT module, adopting a widely tunable MEMS VCSEL, has been assessed in [7]. SSBOFDM is compared to pure DMT transmission over different paths of the ADRENALINE network. The BVT capacity in backto-back (B2B) with optimized BL/PL assignment and $10.5 \mathrm{GHz}$ bandwidth occupancy is above $30 \mathrm{~Gb} / \mathrm{s}$. Bit rates greater than 20 $\mathrm{Gb} / \mathrm{s}$ up to a 2-hop path of $185 \mathrm{~km}$ are supported by adopting OFDM with SSB modulation, due to its higher robustness against chromatic dispersion. In addition, in this case the spectral efficiency is doubled, since only half of the optical bandwidth occupancy is required with respect to the DMT signal. Particularly, the spectral occupancy in terms of assigned flexgrid frequency slots of $12.5 \mathrm{GHz}$ for the case of DMT is two (25 $\mathrm{GHz}$ ), while for the SSB-OFDM slice/flow is only one (12.5 $\mathrm{GHz}$ ).

Table I summarizes the results reported in this section for the different types of VCSEL-based transmitter adopting MCM (either DMT or OFDM) and DD.

\section{AdVANCED FUnCTIONALITIES OF VCSEL-BASED TRANSCEIVERS}

Programmable S-BVTs, relying on an SDN-based control plane paradigm, provide advanced functionalities according to the specific architecture, modules, devices and/or technology adopted for the target design/application.

In this section, we explore the functionalities that can be enabled if the S-BVT is equipped with VCSEL-based module(s). Particularly, we would like to devote special attention to the capability of filling spectral gaps provided by S-BVTs equipped with tunable VCSEL at the transmitter. This feature is particularly relevant to show their potential ability of mitigating the spectrum fragmentation, without requiring a network reoptimization.

Actually, the BVT array of the S-BVT (see Fig. 1) can be composed of subsets of BVT modules, which can also be based on different technologies. This enables a scalable pay-as-yougrow model based on pluggable units and photonic integration. As shown in Fig. 1, the BVTx front-end can be based on either a DM VCSEL or an external modulator driven by a TLS. Each slice generated by the sub-modules of an S-BVT can potentially support a maximum data rate and bandwidth, which can be varied and adapted according to the network channel, target reach and traffic demand. Similarly, by tuning and/or enabling/disabling the laser source(s) as well as the individual subcarrier loading, the optical carrier of each flow can be adapted to the available channel, while suitably allocating and/or squeezing the bandwidth for optimal spectral usage [3]. These features enable rate/distance adaptive functionalities with unique granularity (even finer than EON) and grid adaptation. A soft migration of fixed grid networks towards a flexgrid paradigm is possible, thanks to the use of S-BVTs, enhancing the network flexibility even in fixed-grid networks as demonstrated in [3].

Another important functionality is the slice-ability: multiple adaptive flows are generated/received and aggregated/distributed at the S-BVT. The aggregated flow can be sliced into data flows with less capacity, concurrently serving multiple destination nodes at variable rate over different paths. This functionality also allows enabling inverse multiplexing, as the traffic demand can be split into multiple flows routed via multiple independent paths to the same end-node [3].

\section{A. Spectrum Fragmentation Mitigation Capability}

The reconfigurability of multiple parameters by software defined networking allows the SDN-enabled S-BVT adapting the transmission to the dynamic variation of optical metro networks.

In particular, the suitable configuration of DSP modules, optoelectronic frontends and the aggregator/distributor, also make the S-BVT capable of coping with spectrum fragmentation, as shown in [3]. On one hand, the wavelength tunability and the fine granularity of MCM enable a subcarrier-based spectrum defragmentation; on the other hand, as one or more flow(s) can

TABLE I. PERFORMANCE OF DIRECT MODULATED VCSEL-BASED TRANSCEIVERS ADOPTING MCM AND DD.

\begin{tabular}{|c|c|c|c|c|c|c|c|c|}
\hline \multirow{2}{*}{ MCM } & VCSEL-based Tx & \multirow{2}{*}{ Operating $\boldsymbol{\lambda}$} & \multirow{2}{*}{ 3-dB Bandwidth } & \multicolumn{3}{|c|}{ Performance } & \multirow{2}{*}{ Target Application } & \multirow{2}{*}{ Reference } \\
\cline { 5 - 8 } & & & & Capacity & Link & FEC limit & & \\
\hline DMT & Large-bandwidth VCSEL Tx & $1550 \mathrm{~nm}$ & $15 \mathrm{GHz}$ & $95 \mathrm{~Gb} / \mathrm{s}$ & $4 \mathrm{~km}$ & $1.5 \cdot 10^{-2}$ & Short-reach & {$[8]$} \\
\hline DMT & MEMS-VCSEL Tx & Tunable & $7 \mathrm{GHz}$ & $26 \mathrm{~Gb} / \mathrm{s}$ & $40 \mathrm{~km}$ & $2.26 \cdot 10^{-3}$ & $\begin{array}{c}\text { Converged } \\
\text { WDM-PONs }\end{array}$ & {$[16]$} \\
\hline DMT & VCSEL-based BVT & $1539.61 \mathrm{~nm}$ & $4.5 \mathrm{GHz}$ & $8 \mathrm{~Gb} / \mathrm{s}$ & $200 \mathrm{~km}^{\mathrm{b}}$ & $4.62 \cdot 10^{-3}$ & Metro/Access & {$[17]$} \\
\hline DMT & MEMS-VCSEL (S)-BVT & Tunable ${ }^{\mathrm{c}}$ & $7 \mathrm{GHz}$ & $12 \mathrm{~Gb} / \mathrm{s}$ & $185 \mathrm{~km}^{\mathrm{d}}$ & $4.62 \cdot 10^{-3}$ & Metro Networks & {$[7]$} \\
\hline $\begin{array}{c}\text { SSB- } \\
\text { OFDM }\end{array}$ & MEMS-VCSEL (S)-BVT & Tunable & $7 \mathrm{GHz}$ & $20 \mathrm{~Gb} / \mathrm{s}$ & $185 \mathrm{~km}^{\mathrm{d}}$ & $4.62 \cdot 10^{-3}$ & Metro Networks & {$[7]$} \\
\hline
\end{tabular}


be directed towards the same end-node through different paths, an optimal spectral/resource usage can be performed without requiring a network re-optimization.

When the adaptive DSP is combined with cost-effective and energy-efficient VCSEL-based modules, the programmable transceiver allows suitably filling spectral gaps, thanks to its tunability and adaptive narrow bandwidth. Thus, this technology option results attractive to be explored for mitigating the spectrum fragmentation. Particularly, the adoption of widely tunable MEMS VCSEL configured on-demand by the SDN controller, by means of an SDN S-BVT agent, facilitates this functionality.

In [7], this has been demonstrated for optical metro networks, with fine spectrum granularity. The analysis has been conducted, considering an S-BVT flow/slice generated by a VCSEL-based module in presence of adjacent slices generated by S-BVT modules based on external modulators. In fact, we assume that the S-BVT is composed of subsets of BVT modules as pluggable units, based on either direct or external modulation, equipped with widely-tunable VCSEL or TLS, respectively.

Negligible bitrate penalty has been found for a guard-band of 12.5 GHz between the transmitted channels. In addition, even for lower value of the guard-band, a capacity increase has been evidenced, when the VCSEL module is correctly enabled by the SDN controller. With a guard-band of $6.25 \mathrm{GHz}$, a maximum capacity penalty of less than $10 \%$ is experienced by the VCSEL flow, with respect to the capacity obtained when the adjacent slices are disabled, and less than $30 \%$ penalty is found without any guard-band. Even lower penalties have been measured for the (larger bandwidth) adjacent channels. It has been also assessed that this solution can be used in both flexible and fixedgrid scenarios (soft-migration), even if the latter limits the performance of the S-BVT advanced features [7].

Thus, the proposed S-BVT architecture equipped with such module(s) represents a promising candidate for a hitless spectrum defragmentation, filling spectral gaps with fine granularity and without requiring any network re-optimization.

\section{CONCLUSIONS AND FuturE WORK}

VCSEL technology has been presented as an attractive option to design and implement programmable S-BVT for future agile and high capacity optical metro networks. Starting from previously proposed alternative S-BVT solutions relying on external modulation, the adoption of direct modulated VCSELbased modules has been explored to radically reduce cost, power consumption and footprint. The presented architectures aim to address the challenges of an evolutionary metro network scenario, where a centralized S-BVT of high-capacity (e.g. able to support high peak rate), able to serve multiple endpoints, is shared among distributed (S)-BVTs of lower capacity closer to the end-user.
Recent results have been reported to show the potential of VCSEL technology at $1550 \mathrm{~nm}$ to target metro network distance/capacity. The modularity is key to promote a grow-asneeded paradigm and correctly size the S-BVT to the node/network need, as well as to enable advanced functionalities. Particularly relevant for agile metro network is the ability of VCSEL-based S-BVT modules of filling spectral gaps with fine granularity and coping with the spectrum fragmentation without requiring a network re-optimization.

The proposed architectures based on VCSEL technology, especially if combined with dense photonic integration, represent a very promising solution to achieve ultimate performance in terms of cost/power-efficiency and small footprint. Future work envisions the design and assessment of scalable and modular SBVT architectures able to adaptively generate multiple flows with enhanced capacity to support the high traffic demand of metro networks. The use of large bandwidth VCSELs, to be densely integrated on a same photonic platform, and the adoption of $\mathrm{CO}$ reception will be explored to enhance the S-BVT performance while extending the achievable reach [15]. The programmability and advanced features provided by this approach will be also extensively investigated, towards the integration of these S-BVT architectures in an SDN-based control plane to continue exploring the potential of VCSEL technology.

\section{ACKNOWLEDGMENT}

The authors would like to thank Christian Neumeyr (Vertilas $\mathrm{GmbH}$ ), Alberto Gatto, Paola Parolari and Pierpaolo Boffi (Politecnico di Milano), as well as Juan-Pedro FernándezPalacios (Telefónica I+D) for the fruitful collaboration and discussions that inspire this paper.

\section{REFERENCES}

[1] CISCO White Paper: "The Zettabyte Era: Trends and Analysis," June 2017

[2] M. Svaluto Moreolo, J. M. Fabrega, L. Nadal, F. J. Vilchez, V. López, J. Pedro Fernández-Palacios, "Cost-Effective Data Plane Solutions Based on OFDM Technology for Flexi-Grid Metro Networks Using Sliceable Bandwidth Variable Transponders," Proc. ONDM 2014, Stockholm (Sweden), 19-22 May 2014.

[3] M. Svaluto Moreolo et al., "SDN-Enabled Sliceable BVT Based on Multicarrier Technology for Multiflow Rate/Distance and Grid Adaptation," J. Lightwave Technol., vol. 34, no. 6, pp. 1516-1522, March 2016.

[4] M. Svaluto Moreolo, J. M. Fabrega, L. Martin, K. Christodoulopoulos, E. Varvarigos, J. Pedro Fernández-Palacios, "Flexgrid Technologies Enabling BRAS Centralization in MANs," IEEE/OSA J. Opt. Commun. Netw., vol. 8, no. 7, pp. A64-A75, July 2016.

[5] J. M. Fabrega, M. Svaluto Moreolo, L. Nadal, F. J. Vílchez, R. Casellas, R. Vilalta, R. Martínez, R. Muñoz, J. P. Fernández-Palacios, L. M. Contreras "Experimental Validation of a Converged Metro Architecture for Transparent Mobile Front-/Back-Haul Traffic Delivery using SDN-enabled Sliceable Bitrate Variable Transceivers" IEEE/OSA J. Lightwave Technol., vol. 36, no.7, pp. 1429-1434, Apr. 2018. 
[6] M. Svaluto Moreolo, J. M. Fabrega, and L. Nadal, "S-BVT for nextgeneration optical metro networks: benefits, design and key enabling technologies," in Proc. SPIE 10129, San Francisco, CA (USA), Jan. 2017.

[7] M. Svaluto Moreolo, et al., "Modular SDN-enabled S-BVT Adopting Widely Tunable MEMS VCSEL for Flexible/Elastic Optical Metro Networks," Proc. OFC 2018, S. Diego, CA (USA), March 2018.

[8] C. Xie, P. Dong, S. Randel, D. Pilori, P. Winzer, S. Spiga, B. Kögel, C. Neumeyr, and M.-C. Amann, "Single VCSEL 100-Gb/s short reach system using discrete multi-tone modulation and direct detection," Proc. OFC 2015, paper Tu2H.2, 2015.

[9] C. Xie, S. Spiga, P. Dong, P. Winzer, M. Bergmann, B. Kögel, C. Neumeyr, and M.-C. Amann, "Generation and Transmission of 100-Gb/s PDM 4-PAM Using Directly Modulated VCSELs and Coherent Detection,” Proc. OFC'2014, PDP Th5C.9, 2014.

[10] H. Isono, "Recent standardization activities for client and networking optical transceivers and its future directions," Proc. SPIE 10131, NextGeneration Optical Networks for Data Centers and Short-Reach Links IV, 101310G, Jan. 2017.

[11] X. Zhou, W. Jia, Y. Ma, N. Deng, G. Shen, A. Lord, "An Ultradense Wavelength Switched Network," IEEE/OSA J. Lightwave Technol., vol. 35, no. 11, pp. 2063-2069, 2017.

[12] N. Sambo, et al., "Next generation sliceable bandwidth variable transponder," IEEE Communications Magazine, vol. 53, no. 2, pp. 163171, Feb. 2015.
[13] A. Gatto, D. Argenio, P. Boffi "Very high-capacity short-reach VCSEL systems exploiting multicarrier intensity modulation and direct detection," Optics Express, vol. 24, p. 12769-12775 (2016).

[14] M. Svaluto Moreolo, et al., "Towards Advanced High Capacity and Highly Scalable Software Defined Optical Transmission," Proc. ICTON 2017, Girona (Spain), July 2017.

[15] www.passion-project.eu.

[16] C. Wagner et al., "26-Gb/s DMT Transmission Using Full C-Band Tunable VCSEL for Converged PONs," Photonic technology Lett., vol. 29, no. 17, pp. $1475-1478,2017$.

[17] L. Nadal, et al., "Transparent Service Delivery in Elastic Metro/Access Networks with Cost-Effective Bandwidth Variable Transceivers," Proc. ICTON 2017, Girona (Spain), July 2017.

[18] R. Muñoz, L. Nadal, R. Casellas, M. Svaluto Moreolo, R. Vilalta, J. M. Fabrega, R. Martínez, A. Mayoral, F. J. Vilchez, "The ADRENALINE Testbed: An SDN/NFV Packet/Optical Transport Network and Edge/Core Cloud Platform for End-to-End 5G and IoT Services," in Proc. EuCNC 2017, Jun. 2017

[19] S. Pau et al., "10-Gb/s direct modulation of widely tunable $1550-\mathrm{nm}$ VCSEL," J. Selected Topics Quantum Electron., vol. 21, no. 6, p. 1700908 , Nov/Dec. 2015. 